\title{
Dopamine Transporter and Reward Anticipation in a Dimensional Perspective: A Multimodal Brain Imaging Study
}

\begin{abstract}
Manon Dubol', Christian Trichard ${ }^{1,2}$, Claire Leroy ${ }^{1,3}$, Anca-Larisa Sandu ${ }^{1,4}$, Mehdi Rahim ${ }^{5}$, Bernard Granger ${ }^{1,6}$, Eleni T Tzavara ${ }^{1,6,7}$, Laurent Karila',8, Jean-Luc Martinot' ${ }^{1}$ and Eric Artiges ${ }^{*, 1,9}$

'INSERM, Research Unit 1000 'Neuroimaging and Psychiatry', Paris Sud University_Paris Saclay University, Paris Descartes University, Maison de Solenn, Paris \& Service Hospitalier Frédéric Joliot, Orsay, France; ${ }^{2}$ EPS Barthelemy Durand, Etampes, France; ${ }^{3}$ Laboratoire Imagerie Moléculaire In Vivo (IMIV), CEA, INSERM, CNRS, Paris Sud University_Paris Saclay University, Orsay, France; ${ }^{4}$ Aberdeen Biomedical Imaging Centre, University of Aberdeen, Foresterhill, Aberdeen, UK; ${ }^{5}$ Parietal Project Team—INRIA, CEA, Neurospin, Gif-Sur-Yvette, France; ${ }^{6}$ Tarnier Psychiatry Department, AP-HP, Cochin Hospital, Paris, France; ' INSERM UII 30 Research Unit, CNRS UMR 8246, UPMC UM CR I 8, Paris, France; ${ }^{8}$ AP-HP, Addiction Research and Treatment Center, Paul Brousse Hospital, Villejuif, France; ${ }^{9}$ Groupe Hospitalier Nord Essonne, Psychiatry Department, Orsay, France
\end{abstract}

Dopamine function and reward processing are highly interrelated and involve common brain regions afferent to the nucleus accumbens, within the mesolimbic pathway. Although dopamine function and reward system neural activity are impaired in most psychiatric disorders, it is unknown whether alterations in the dopamine system underlie variations in reward processing across a continuum encompassing health and these disorders. We explored the relationship between dopamine function and neural activity during reward anticipation in 27 participants including healthy volunteers and psychiatric patients with schizophrenia, depression, or cocaine addiction, using functional magnetic resonance imaging ( $\mathrm{fMRI}$ ) and positron emission tomography (PET) multimodal imaging with a voxel-based statistical approach. Dopamine transporter (DAT) availability was assessed with PET and [ ' C]PE2I as a marker of presynaptic dopamine function, and rewardrelated neural response was assessed using $\mathrm{MMRI}$ with a modified Monetary Incentive Delay task. Across all the participants, DAT availability in the midbrain correlated positively with the neural response to anticipation of reward in the nucleus accumbens. Moreover, this relationship was conserved in each clinical subgroup, despite the heterogeneity of mental illnesses examined. For the first time, a direct link between DAT availability and reward anticipation was detected within the mesolimbic pathway in healthy and psychiatric participants, and suggests that dopaminergic dysfunction is a common mechanism underlying the alterations of reward processing observed in patients across diagnostic categories. The findings support the use of a dimensional approach in psychiatry, as promoted by the Research Domain Criteria project to identify neurobiological signatures of core dysfunctions underling mental illnesses.

Neuropsychopharmacology (2018) 43, 820-827; doi:I0.1038/npp.2017.183; published online 20 September 2017

\section{INTRODUCTION}

Regional dysfunctions of the dopamine (DA) system and reward-related neural activity have both been reported in various psychiatric disorders (Davis et al, 1991; Chau et al, 2004; Hommer et al, 2011; Fusar-Poli and Meyer-Lindenberg, 2013). Although animal studies have established a close relationship between DA and reward using neurophysiological paradigms (Schultz, 1997; Carelli and Wightman, 2004), direct evidence for their covariation in humans remains limited. Particularly, it is unknown whether gradual alterations in the DA system underlie variations in reward processing across a continuum encompassing health and psychiatric disorders.

*Correspondence: Dr E Artiges, INSERM UI000, Service Hospitalier Frédéric Joliot, 4 Place Général Leclerc, Orsay 9|40।, France, Tel: +(33) | 698678 23, Fax: +(33) | 698678 10, E-mail: eric.artiges@inserm.fr Received 10 May 2017; revised 9 August 2017; accepted 13 August 2017; accepted article preview online 22 August 2017
In healthy subjects, positron emission tomography (PET) and functional magnetic resonance imaging (fMRI) have been recently used to explore the relationship between DA release and reward-related functional activity. Weiland et al $(2014,2016)$ described positive correlations between DA release within the nucleus accumbens (NAcc), assessed with $\left[{ }^{11} \mathrm{C}\right]$ raclopride, and reward-related activations in the prefrontal cortex and NAcc. Similarly, another team showed significant positive correlations between DA release in the NAcc and both midbrain and NAcc activations during reward anticipation (Schott et al, 2008). The local correlation between DA release and neural activity during anticipation of reward in the NAcc has also been observed using PET and $\left[{ }^{18} \mathrm{~F}\right]$ fallypride (Buckholtz et al, 2010). Otherwise, using PET and $\left[{ }^{18} \mathrm{~F}\right]$-FDOPA, correlations between dopamine synthesis capacity within the midbrain and reward anticipation and feedback in the prefrontal cortex were also reported (Dreher et al, 2008). Thus, PET-fMRI multimodal imaging seems appropriate to assess the relationship between the DA system 
and the reward system from healthy to pathological conditions.

Abnormalities of the reward system and the mesolimbic DA system have been reported separately in patients with psychiatric disorders, including schizophrenia, depression, and addictions (Chau et al, 2004). Striatal presynaptic DA hyperactivity (Fusar-Poli and Meyer-Lindenberg, 2013) is central to the DA hypotheses of schizophrenia (Davis et al, 1991; Kapur, 2003). It has been proposed that DA hyperactivity in schizophrenia patients would result in an aberrant attribution of salience to irrelevant stimuli (Kapur, 2003), then contributing to abnormal association with reward. In major depressive disorder, a reduced striatal response to rewards (Whitton et al, 2015) has been hypothesized to relate to the loss of pleasure and motivation found in these patients (Naranjo et al, 2001). Moreover, the monoamine deficiency hypothesis posits that depressive symptoms arise from insufficient levels of serotonin, norepinephrine, but also DA (Delgado, 2006). In addicted patients, the magnitude of striatal DA release following drug intake is predictive of the subjective effects of the drug (Malison et al, 1995; Volkow et al, 1997). Volkow et al (2011) also highlighted the involvement of striatal DA in drug craving in cocaine-dependent subjects. Further, chronic drug users display a downregulation of DA and reward systems, attested by lower D2 availability and reward-related activations (Hommer et al, 2011).

Among markers of the DA system, the dopamine transporter (DAT), which has a key role in synaptic DA regulation, has been proposed to reflect DA system integrity and function (McHugh and Buckley, 2015). Moreover, though controversial, DAT modifications have been reported in addictions (Volkow et al, 2004; Narendran and Martinez, 2008; Leroy et al, 2012; Hirth et al, 2016), mood disorders (Pinsonneault et al, 2011), and schizophrenia (Sjoholm et al, 2004; Arakawa et al, 2009; Artiges et al, 2017). Thus, assessing DAT availability to explore the relationship between DA and reward processing in psychiatric populations appears particularly relevant.

In regard to the assessment of the reward system, the Monetary Incentive Delay (MID) task (Knutson et al, 2000) has emerged as the most reliable and the most widely used tool to measure activations related to reward anticipation and feedback in fMRI. Indeed, the MID task revealed that anticipation of increasing amounts of monetary reward is related to NAcc activation (Knutson et al, 2001a), whereas reward feedback is associated with activations within the ventromedial frontal cortex (Knutson et al, 2001b). Furthermore, abnormalities of the reward system activity have been reported in patients with addiction, schizophrenia and major depression using the MID task (Hagele et al, 2015).

With the establishment of the Research Domain Criteria project (RDoC), the National Institute of Mental Health (NIMH) promotes the use of a dimensional approach that can be applied across diagnostic boundaries to identify neurobiological signatures of core dysfunctions underling mental illnesses (Insel et al, 2010; Hagele et al, 2015). Thus, measuring DA and reward systems in this context might clarify the complex relationships between DA at the molecular scale and DA-related functional responses across clinical conditions.
In line with this dimensional approach, we hypothesized that DAT availability would correlate with reward anticipation-related neural response in a group of participants including healthy controls and patients with schizophrenia, depression, or cocaine-dependence. We assumed that DA and reward systems remain strongly associated in psychiatric patients so that abnormal DA function should be related to abnormal reward processing. To this end, we used $\left[{ }^{11} \mathrm{C}\right]$ PE2I PET that assesses DAT availability, and fMRI with a modified version of the MID task that assesses reward processing, in voxel-based correlational analyses. In addition to the dimensional approach, we examined the relationship between DAT and reward-related fMRI activations within each clinical subgroup, in a secondary exploratory analysis.

\section{MATERIALS AND METHODS}

PET and $\mathrm{AMRI}$ investigations were approved by the regional biomedical research ethics committee (CPP Ile de France 7), and each participant gave written informed consent after receiving full information on the procedures.

\section{Participants}

In this study, we used PET images acquired with $\left[{ }^{11} \mathrm{C}\right] \mathrm{PE} 2 \mathrm{I}$ in healthy and psychiatric samples, on which previous PET studies investigating DAT were based (Leroy et al, 2012; Karila et al, 2016; Artiges et al, 2017). From this database, twenty-seven participants were selected based on the combined presence of PET images and fMRI data acquired with the MID task, that passed quality control (Supplementary Figure S1). Our sample included six healthy controls (HC); ten cocaine-dependent patients that were abstinent for at least 3 days (COC); six patients with schizophrenia (SCZ) and five depressive patients (DEP). SCZ and DEP met criteria for a diagnosis of schizophrenia or major depressive disorder according to the DSM-IV-TR and were recruited by senior psychiatrists from psychiatric departments of Central and South Paris area. COC met DSM-IV criteria for cocaine dependence and were recruited in the Cocaine Reference Center within Paul Brousse University Hospital, Villejuif (France). In this sample, SCZ were administered atypical antipsychotics in monotherapy, DEP were treated with Selective Serotonin Reuptake Inhibitors (SSRI) monotherapy (sertraline excluded), and COC were untreated. Urinary toxicology screening tests were carried out using benzoylecgonine (BE) dosage to ensure cocaine abstinence in COC patients. HC were recruited from the community through billboards in Paris area.

Exclusion criteria were: age over 60, any substance related Axis I disorder in the past 6 months (except tobacco dependence for all participants and cocaine dependence for COC subgroup), treatment susceptible to interfere directly with DAT, electroconvulsive therapy treatment in the past six months, history of epileptic seizures, other psychiatric and/or neurological disorders or substantial brain damage, and contraindication to magnetic fields according to established safety criteria. Given the known action of psychoactive drugs on the DA system, semi-quantitative urinary multi-screens for the detection of cocaine, amphetamine, methamphetamine, cannabis, methadone, opiates, 
ecstasy, barbiturates, benzodiazepines, and tricyclic antidepressants (BMD: Biomedical Diagnostics) were performed prior to each imaging session in order to rule out multiple drug users.

\section{Task Description}

During fMRI scanning, all participants completed the modified Monetary Incentive Delay task (MID-task) reward paradigm (Figure 1), in which the main difference to the original version is the omission of loss trials (Knutson et al, 2000; Nees et al, 2012).

\section{MRI Acquisition}

Structural and functional MRI were acquired using a 1.5 Tesla whole-body system (Signa, General Electric, Milwaukee, Wisconsin). T1-weighted structural MRI scan was carried out with the following parameters: 3D Fouriertransform spoiled-gradient-recalled acquisition with $\mathrm{TR}=12.5 \mathrm{~ms}, \mathrm{TE}=2.2 \mathrm{~ms}, 124$ contiguous slices, $256 \times 256$ view matrix, voxel size $=0.9375 \times 0.9375 \times 1.3 \mathrm{~mm}$.

For functional MRI, 66 randomized trials were presented and 290 volumes were acquired for a total duration of $11 \mathrm{~min}$ (Figure 1). Stimuli were presented through mirror glasses and an active matrix video projector. We acquired 36 slices in ascending order using a gradient-echo $\mathrm{T} 2{ }^{\star}$-weighted sequence and the following image parameters: $\mathrm{TR}=2,400 \mathrm{~ms}, \mathrm{TE}=30 \mathrm{~ms}$, and an inplane matrix size of $64 \times 64$ pixels. Voxels size was $3.75 \times 3.75 \times 3 \mathrm{~mm}$.

\section{fMRI Processing}

The fMRI data were processed using Statistical Parametric Mapping (SPM12, Wellcome Department of Imaging Neuroscience, University College London, London, UK). Images were corrected for slice timing, spatially realigned, warped onto the MNI space and smoothed using a $10 \mathrm{~mm}$ FWHM Gaussian filter. First-level analysis of blood oxygen level-dependent (BOLD) signal change was performed by modeling anticipation and feedback as explanatory variables within a general linear model for each subject. We defined reward magnitudes of large, small and no win as subjectspecific variables of interest, and movement regressors were added to the design matrix. To focus on neural response during reward anticipation, we used the 'anticipation of large win $v s$ small win' contrast in second-level analyses.

\section{PET Acquisition and Processing}

PET imaging was performed on a Siemens ECAT HRRT 3DPET scanner (CPS innovations Services, Knoxville, TN, USA) using [ $\left.{ }^{11} \mathrm{C}\right]$ PE2I tracer, a potent DAT radioligand that binds with high affinity (Ki: $17 \mathrm{nM}$ in vitro), specificity and selectivity to central DAT (Halldin et al, 2003). The radiotracer was prepared using a TRACERlab FX-C Pro synthesizer (Gems, Velisy, France). The PET acquisition started with the bolus injection of $300 \mathrm{MBq}$ of $\left[{ }^{11} \mathrm{C}\right] \mathrm{PE} 2 \mathrm{I}$ and lasted $60 \mathrm{~min}$ (acquisition of 20 sequential frames from 1 to $5 \mathrm{~min})$. Images were reconstructed using the ordinary Poisson-ordered subset expectation maximization (OPOSEM) algorithm with Point Spread Function (PSF)
Modified Incentive Delay Task (MID)

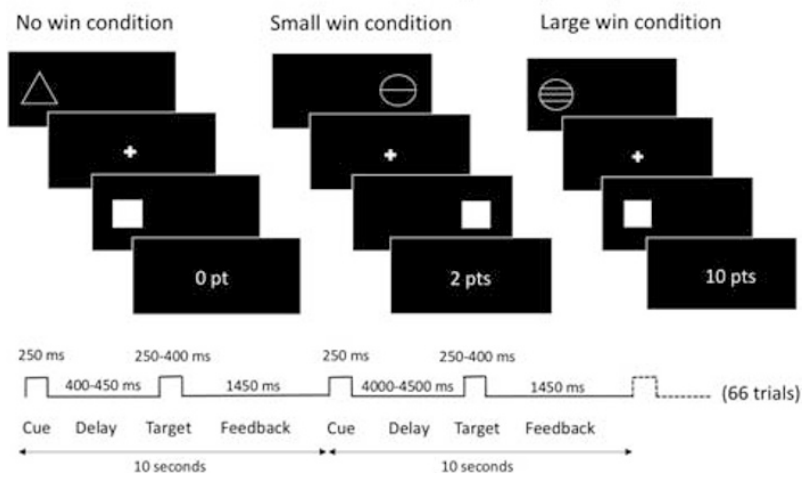

Figure I Modified Monetary Incentive Delay task participants have to respond as quickly as possible with left or right index finger to hit a target (white square) that appears for a short time on the left or right side of the screen. When subjects hit the target in time on the appropriate side, they score points. A preceding clue provides information on the points to be won. A triangle indicates no points, a circle with one line, two points, and a circle with three lines, ten points. For each condition (no win, small win, and big win), 22 trials were presented in equal proportion for each target side. A short training period outside the scanner was carried out to ensure a full understanding of the task.

modeling. The voxel size was $1.2 \times 1.2 \times 1.2 \mathrm{~mm}$. The injected radioactivity was $293.71 \pm 55.31 \mathrm{MBq}$ and the specific radioactivity was $32.13 \pm 17.10 \mathrm{GBq} / \mu \mathrm{mol}$.

Head motion corrections were carried out postreconstruction using frame by frame co-registration of the PET dynamic series with a reference frame presenting a high $\left[{ }^{11} \mathrm{C}\right]$ PE2I uptake and a mutual information method within the BrainVISA/ Anatomist software (http://brainvisa.info). Thereafter, brain regions were determined by T1-MRI automatic parcellation and applied on dynamic coregistered PET images using the PNEURO tool of PMOD imaging software (Version 3.4, PMOD Technologies Ltd, Zürich, Switzerland), to process parametric binding potential images. Time-activity curves obtained from bilateral dorsal caudate and putamen nuclei as high-specific binding and crus1 sub-region of the cerebellum as a reference tissue that display non-specific binding were exported to PMOD's pixel-wise tool. Parametric maps of the regional $\left[{ }^{11} \mathrm{C}\right] \mathrm{PE} 2 \mathrm{I}$ non-displaceable binding potential $\left(\mathrm{BP}_{\mathrm{ND}}\right)$ were generated using Gunn's basis function method (Gunn et al, 1997), which is closely related to the simplified reference tissue model (SRTM) (Lammertsma and Hume, 1996). The suitability of specific $\left[{ }^{11} \mathrm{C}\right] \mathrm{PE} 2 \mathrm{I}$ binding quantification using a compartmental approach and the cerebellum as reference region has been confirmed by previous works (Seki et al, 2010). Spatial normalization was applied on the $\mathrm{BP}_{\mathrm{ND}}$ maps using SPM8 with a ligand-specific $\left[{ }^{11} \mathrm{C}\right] \mathrm{PE} 2 \mathrm{I}$ template generated according to an MRI-aided procedure. The normalized $\mathrm{BP}_{\mathrm{ND}}$ maps were smoothed using a $10-\mathrm{mm}$ FWHM Gaussian filter. The voxel size was $2 \times 2 \times 2 \mathrm{~mm}$.

\section{PET Statistical Analysis}

As previously described (Karila et al, 2016; Artiges et al, 2017), we used a mask for the PET statistical analysis, in order to include only main DA regions. It included basal ganglia, insula, amygdala, thalamus, midbrain, anterior cingulate gyrus, inferior frontal 
cortex, and temporal cortex (hippocampus, parahippocampal gyrus, superior temporal gyrus).

To enable PET-fMRI correlations, we performed $\mathrm{BP}_{\mathrm{ND}}$ values extraction from a one-sample $t$-test of PET images, using the MarsBaR toolbox implemented in SPM. Thus, individual averaged raw $\mathrm{BP}_{\mathrm{ND}}$ values were extracted within specific anatomical regions of interest (ROIs) of the DA system. The ROIs included midbrain (substantia nigra (SN) and ventral tegmental area (VTA)), NAcc and dorsal striatum (dorsal caudate and dorsal putamen nuclei). As the spatial resolution of PET images does not allow the distinction between SN and VTA, we used one midbrain ROI encompassing the two structures.

\section{PET-fMRI Correlation}

In order to examine the relationships between DAT availability and the reward system neural response, we performed a PET-fMRI multimodal analysis. Mean $\mathrm{BP}_{\mathrm{ND}}$ values extracted from each previously defined ROI were included as covariate of interest in voxel-based correlation analyses over the fMRI contrast maps of reward anticipation 'large win vs small win'. For each regional mean $\mathrm{BP}_{\mathrm{ND}}$, we performed a multiple regression analysis in SPM12. Age, clinical status, and tobacco use were included as confounding covariates, due to their potential effects on both DAT levels and functional activations. For these multimodal correlation analyses, height threshold was set at $p<0.05$ family-wise error (FWE)-corrected and cluster significance (extent threshold) was set at 10 voxels.

Multimodal correlation statistics were conducted within the mask of PET analysis, which includes mainly dopaminergic brain regions (Karila et al, 2016). This mask allowed the exclusion of premotor cortex activations occurring during reward anticipation periods preceding the motor response. As a control, we have tested the correlation between premotor cortex activations and DAT availability in the defined ROIs and no association was found (data not shown).

\section{Secondary Exploratory Analyses}

To further explore the results obtained from the PET-fMRI correlation, we ran a general linear model in a post hoc analysis using extracted $\mathrm{BP}_{\mathrm{ND}}$ and activations raw data with JMP10 software (JMP, SAS Institute). Functional activation values were extracted from significant activated clusters of the fMRI contrast map 'anticipation of large win vs small win' using the MarsBaR toolbox in SPM. We included reward anticipation activations as independent variable and regional $\mathrm{BP}_{\mathrm{ND}}$ values, age, tobacco use, and clinical subgroups as dependent variables of the model. We tested for the main effects of each dependent variable and for the subgroup ${ }^{\star} \mathrm{BP}_{\mathrm{ND}}$ interaction. Thereafter, we assessed the PET-fMRI correlation within each clinical subgroup separately in exploratory analyses, using a similar statistical model.

\section{RESULTS}

\section{Participants' Characteristics}

Subgroups of participants did not differ by age (mean $=$ $\left.36.52 \pm 8.00, \chi^{2}=1.80, p=0.61\right)$, body mass index $(\mathrm{BMI})$ (mean $\left.=25.86 \pm 4.06, \chi^{2}=4.67, p=0.20\right)$ and daily tobacco consumption $\left(\right.$ mean $\left.=13.57 \pm 11.12, \chi^{2}=3.05, p=0.38\right)$, as revealed by Kruskal-Wallis rank tests performed across the four clinical categories.

\section{Behavioral Results}

During the MID task, the participants displayed high rates of correct responses, on an average of $86.50 \%$ mean success. ANOVA and post-hoc analyses of hit and miss rates under the three conditions (large, small, and no win) revealed a significant effect of incentive conditions ( $F=7.69$, $p=0.0009$ ), with lower performance in unrewarded trials compared to rewarded trials (large win $v s$ no win: $p=0.0008$, small win $v s$ no win: $p=0.015)$.

\section{fMRI Results}

The reward anticipation contrast map 'large win $v s$ small win' revealed specific activations in ventral parts of caudate and putamen nuclei, insula, anterior and mid cingulate cortices, inferior frontal cortex, premotor cortex, and supplementary motor area (Supplementary Table S1), as initially described by Knutson et al (2000, 2001a, 2001b) with the original MID task in healthy subjects.

\section{PET Results}

In the whole group, significant binding of $\left[{ }^{11} \mathrm{C}\right]$ PE2I was detected within the striatum (caudate and putamen), pallidum, insula, thalamus, hippocampus, and midbrain, as determined with the Montreal Neurological Institute (MNI) coordinates, from the local maxima of each region of the cluster (Supplementary Figure S2; Supplementary Table S2).

\section{PET-fMRI Correlation}

Across all participants, DAT availability in the midbrain correlated positively with anticipatory reward neural response in bilateral NAcc ([MNI coordinates $x, y, z]:[-8,16$, $-10], \quad t=8.61, \quad p_{\mathrm{FWE}}=0.0002 ; \quad[8,16,-12], \quad t=6.02$, $\left.p_{\text {FWE }}=0.015\right)$ and in the left inferior/orbital prefrontal cortex $\left([-24,26,-8], t=5.92, p_{\mathrm{FWE}}=0.018\right)$; Figure 2 . There was no significant correlation between $\mathrm{BP}_{\mathrm{ND}}$ values in the dorsal striatum and reward-related activations.

\section{Secondary Exploratory Analyses}

A secondary analysis conducted on extracted fMRI contrast estimates confirmed that $\mathrm{BP}_{\mathrm{ND}}$ values in the midbrain exert a significant influence on reward-related functional neural response in the ventral striatum cluster (likelihood ratio $=$ 14.99, $p<0.0001)$. Similarly, we found significant effects of age (positive effect, likelihood ratio $=25.79, p<0.0001$ ), daily tobacco consumption (negative effect, likelihood ratio $=9.98$, $p=0.0016$ ), and subgroups (likelihood ratio $=29.93$, $p<0.0001)$ on fMRI contrast estimates, indicating a significant influence of these factors on NAcc reward-related neural response. Otherwise, there was no interaction between 'subgroup' and 'midbrain $\mathrm{BP}_{\mathrm{ND}}$ ' factors (likelihood ratio= 5.51, $p=0.1379$ ), suggesting that the correlation between midbrain $\mathrm{BP}_{\mathrm{ND}}$ values and the neural response to reward 
a PE2I BP ${ }_{\text {ND }}$ map
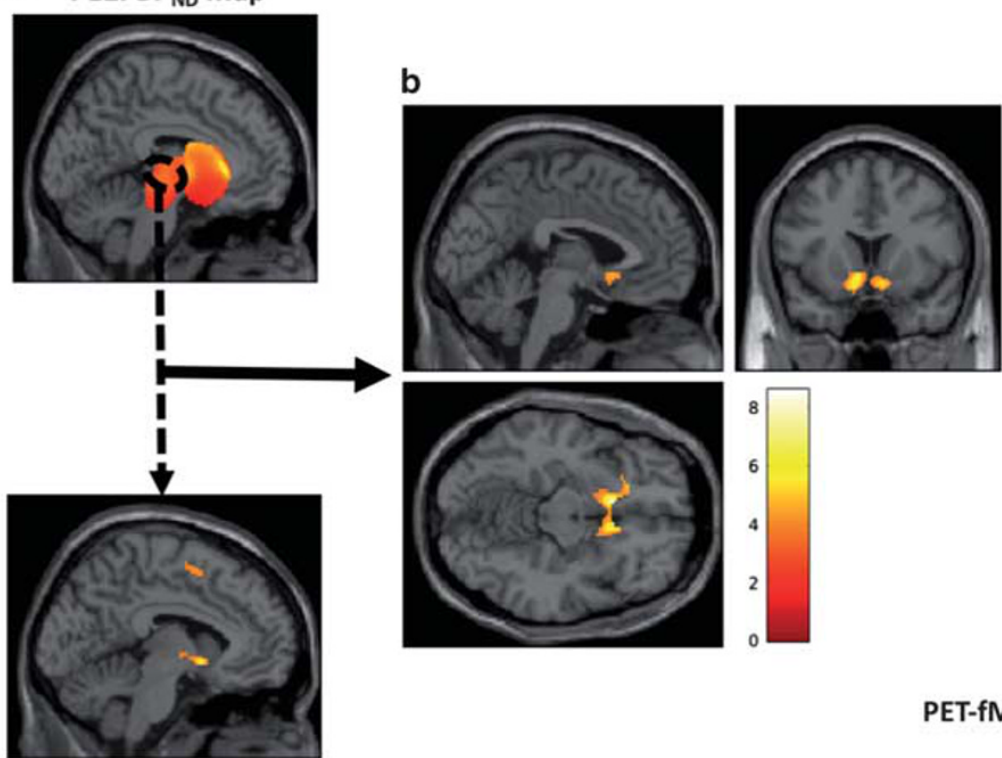

C

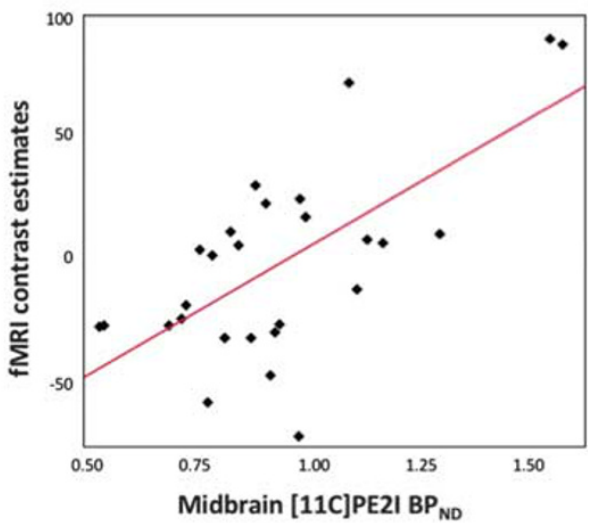

PET-fMRI correlation

fMRI contrast map

" anticipation large vs small win »

Figure 2 Correlation between midbrain dopamine transporter (DAT) availability and anticipatory reward activations. (a) Slice views of [ ' C]PE2I nondisplaceable binding potential $\left(\mathrm{BP}_{\mathrm{ND}}\right.$ ) maps with delineated midbrain region of interest where $\mathrm{BP}_{\mathrm{ND}}$ values were extracted (upper), and fMRI contrast map of reward anticipation 'large win vs small win' (lower). (b) Slice view of the correlated cluster (388 voxels mainly including nucleus accumbens) overlaid onto a MRI template. For presentation purpose, we used a significance threshold set at $0.00 \mathrm{I}$ uncorrected for voxel level and an extent threshold set at 0.05 familywise error (FWE)-corrected for multiple comparisons. (c) Plot of bivariate fit in the nucleus accumbens peak voxel [MNI coordinates -8 , I6, - I0], $P_{F W E}=0.0002$.

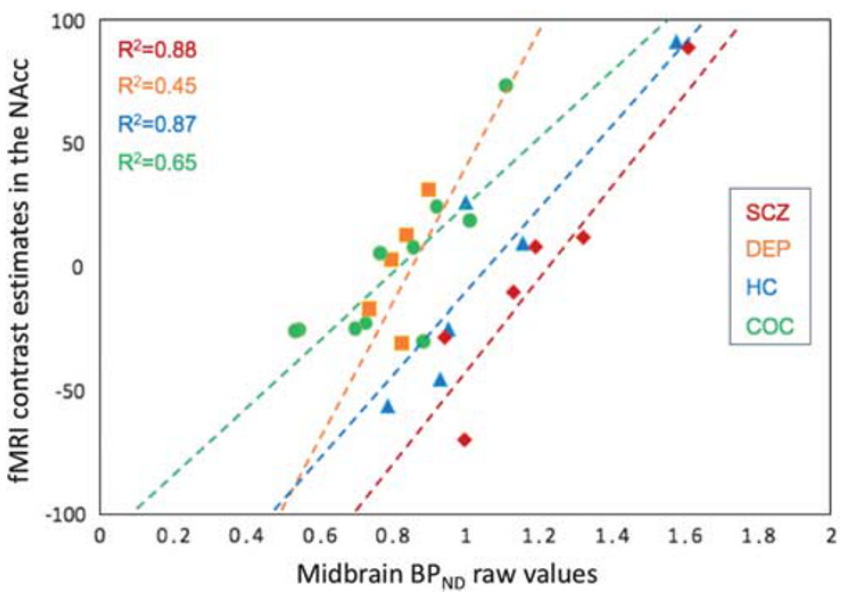

Figure 3 PET-FMRI correlation within each subgroup. Correlation graph derived from general linear model analyses conducted within each subgroup and including functional MRI contrast 'anticipation of large win vs small win' estimates in the nucleus accumbens as exploratory variable and midbrain [ I'C]PE2I non-displaceable binding potential (BPND) as explanatory variable.

anticipation in the NAcc did not differ according to the clinical condition of the participants. When testing this correlation within each subgroup separately for exploratory analyses, we replicated our main result showing the influence of $\mathrm{BP}_{\mathrm{ND}}$ values on fMRI contrast estimates (Figure 3) in $\mathrm{HC}$ (likelihood ratio $=12.11, p=0.0005$ ), COC (likelihood ratio $=$ $11.40, p=0.0007$ ), SCZ (likelihood ratio $=23.23, p<0.0001$ ), and DEP (likelihood ratio $=6.20, p=0.0128$ ), despite the small sample sizes.

\section{DISCUSSION}

This is the first direct evidence of a relationship between DAT availability in the midbrain and BOLD activity within the NAcc and the left inferior/orbital prefrontal cortex during reward anticipation in healthy controls and psychiatric patients.

This finding is in line with previous multimodal PET-fMRI studies that established a link between reward function and DA release in healthy humans. Using PET with $\left[{ }^{11} \mathrm{C}\right]$ raclopride or $\left[{ }^{18} \mathrm{~F}\right]$ fallypride, and fMRI of reward anticipation, correlations between DA release in the NAcc and both midbrain and NAcc activations have been described (Schott et al, 2008; Buckholtz et al, 2010; Weiland et al, 2016). Consistently with Schott et al (2008) report of a relationship between DA binding on D2 receptors in the NAcc and functional activations in the midbrain, we report a correlation linking the DAT in the midbrain to the NAcc neural response, which associates the two main anatomically interconnected regions of the mesolimbic system. Moreover, the correlation between DAT availability in the midbrain and anticipatory reward response also included the left inferior/ orbital prefrontal cortex that receives a large amount of midbrain DA outputs from the mesocorticolimbic pathway, together with the NAcc and the anterior cingulate cortex (Haber and Behrens, 2014). In the other way, this region provides a large part of the cortical afferent projection to the NAcc (Haber and Behrens, 2014). These interconnections between the inferior/orbital prefrontal cortex, the NAcc and the midbrain are essential to the function of the reward system and underlie the consistency of our results. 
Surprisingly, the local correlation between DA and rewardrelated activations within the NAcc reported in the previous studies was not replicated in our sample. This could be explained by the difference between the DA markers used, since our study explored DAT availability and previous works assessed DA receptors availability or DA release. However, when taking a more lenient extent threshold, we found a local positive correlation between DAT availability and anticipatory reward response within the left NAcc, $([-8$, $\left.18,-10], t=6.17, p_{\mathrm{FWE}}=0.011\right)$. As the DAT represents a useful marker of the DA system integrity and function (McHugh and Buckley, 2015), and the NAcc is one of the main targets of DA neurons located in the midbrain, the correlations we found between DAT availability in the midbrain or in the NAcc and functional activity within the NAcc adds evidence for a modulatory role of DA in reward anticipation (Ikemoto, 2007).

In addition, there is also indirect evidence of a relationship between DAT and reward processing, which emerged from studies that explored the links between dopaminergic gene variants and NAcc reward responsivity in healthy volunteers (Dreher et al, 2009; Camara et al, 2010; Greer et al, 2016), supporting the relevance of assessing DAT in a context of reward processing.

Since the relationship between DA and reward is well established in animals (Schultz, 1997; Carelli and Wightman, 2004), we hypothesized that the DA function and the reward system neural activity remain strongly associated in humans with psychiatric disorders where dysfunctions of both systems were reported (Davis et al, 1991; Chau et al, 2004; Hommer et al, 2011; Fusar-Poli and Meyer-Lindenberg, 2013), so that abnormal DA function should be associated to abnormal reward processing. Consistently with this hypothesis, we found no interaction between clinical subgroup and midbrain $\mathrm{BP}_{\mathrm{ND}}$ on NAcc reward-related activations, suggesting that the relationship between DA function and reward anticipation does not differ according to the clinical subgroups. Remarkably, the DAT availability and reward anticipation measures vary similarly across the participants, so that the clinical subgroups with the lowest DAT levels display the lowest functional activations. Although observed in small samples of participants with various conditions, this original finding further supports the interest of a dimensional approach of the DA regulation as a modulator of the reward system.

By merging different psychiatric patients and healthy controls, we obtained a large variability in PET and fMRI measures that can be described as a continuum through the clinical subgroups. In the context of the RDoC project, it has been proposed that a dimensional approach can be used across clinical categories to identify the pathophysiology of core dysfunctions found in mental illnesses (Insel et al, 2010). In the present study, despite the heterogeneity of psychiatric disorders examined, the relationship between DA and reward is conserved regardless of the condition of the participants. Besides, we replicated our main result in healthy controls and each patient subgroup separately. Although the interpretation of this exploratory analysis is limited by the number of participants in each subgroup, the results suggest that dopaminergic dysfunction is a common mechanism underlying the alterations of reward processing observed in patients with schizophrenia, depression, or addiction. Most importantly, these data demonstrate the relevance of studying the physiopathology of psychiatric disorders in a dimensional perspective.

Some limitations should be highlighted. First, although the cohort size is in line with previous studies that combined PET and fMRI imaging (Schott et al, 2008; Weiland et al, 2014; Weiland et al, 2016), the small subgroup sizes do not enable robust intergroup comparisons and imply that subgroup results should be considered with caution. Second, as the effects of medication and groups cannot be disentangled in the statistical analyses, their putative impact need to be addressed in future studies. SCZ were treated with atypical antipsychotics that were shown to have no effect on the DAT (Lavalaye et al, 2000; Kim et al, 2004; Artiges et al, 2017) or on activation patterns to monetary reward in ventral striatum (Juckel et al, 2006; Walter et al, 2009; Nielsen et al, 2012). DEP were treated with SSRI. Although SRRIs do not bind tightly to DAT (Zhou et al, 2009), they were shown to induce an up-regulation of the DAT protein (Chen and Lawrence, 2003; Kugaya et al, 2003; Rominger et al, 2015). Thus, it cannot be excluded that SSRI treatment may have slightly influenced DAT quantification in depressed patients. Regarding reward processing, whether SSRI treatment influences functional activity during reward tasks in patients with depression remains unclear. In healthy controls, SSRI treatment was shown to reduce the responsiveness to reward in fMRI (Macoveanu et al, 2014; Graf et al, 2016). However, Stoy et al (2012) evidenced a hyporeactivity to reward in unmedicated depressed patients, which is normalized after successful SSRI treatment. Overall, the potential impacts of medications on PET and fMRI measures could be considered as a limitation of the study. Besides, although the $\left[{ }^{11} \mathrm{C}\right] \mathrm{PE} 2 \mathrm{I}$ binds with high affinity to the DAT and is about $120-145 \times$ more potent that DA itself (Reith et al, 1996), an effect of endogenous DA on DAT binding cannot be excluded. Another limitation arises from the differences between the behavioral and clinical features assessed in the studies that form our database, thus precluding dimensional correlations between imaging data and psycho-behavioral measures.

The combined use of PET and fMRI allows the assessment of DA and reward neural networks from molecular to functional levels. The present results demonstrate for the first time that DAT availability in the midbrain, which contains the cell bodies of dopaminergic neurons, correlates with BOLD activity during reward anticipation in the NAcc, the main DA target within the mesolimbic pathway. This relationship between DA and reward systems transcends diagnostic categories in psychiatric patients, which highlights the interest of studying such systems in patients with a dimensional approach. The use of multimodal and multiscale assessments in a dimensional perspective could give an overall view of molecular, structural and functional alterations that underlie the pathophysiology of psychiatric disorders in future research and could lead to the development of new treatment strategies thereafter.

\section{FUNDING AND DISCLOSURE}

Imaging acquisitions and support to A-LS were funded by grants from the National Agency for Research (ANR) (APV05143LSA); the National Institute of Health and 
Medical Research (INSERM); the Interministerial Mission in the Fight Against Drugs and Drug Addiction (MILDT, Grant No. AO2004-37); the French Ministry of Health (PHRC 2007) and from MILDECA-INCa-INSERM (2006). MD is supported by the «Investissement d'Avenir» program of the French Ministry of Education and Research, through the Lidex-PIM project funded by the IDEX Paris-Saclay. ETT was supported by a join INSERM-APHP grant. The authors declare no conflict of interest.

\section{ACKNOWLEDGMENTS}

We would like to thank Christine Baron, Vincent Brulon, Stéphane LeHelleix, Stéphane Demphel, Claude Comtat, Frédéric Dollé, Philippe Gervais, and Renaud Maroy from the Service Hospitalier Frédéric Joliot for their efficient technical support and ${ }^{11} \mathrm{C}$ radioligand preparation. They thank Marie Prat, Audrey Pepin, and Audrey Mabondo for their help in PET processing and Pr. Maria-Joao SantiagoRibeiro and Dr Renaud de Beaurepaire for their involvement in the recruitment of participants.

\section{REFERENCES}

Arakawa R, Ichimiya T, Ito $\mathrm{H}$, Takano A, Okumura M, Takahashi $\mathrm{H}$ et al (2009). Increase in thalamic binding of [(11)C]PE2I in patients with schizophrenia: a positron emission tomography study of dopamine transporter. J Psychiatr Res 43: 1219-1223.

Artiges E, Leroy C, Dubol M, Prat M, Pepin A, Mabondo A et al (2017). Striatal and extrastriatal dopamine transporter availability in schizophrenia and its clinical correlates: a voxel-based and high-resolution PET study. Schizophr Bull (e-pub ahead of print).

Buckholtz JW, Treadway MT, Cowan RL, Woodward ND, Benning $\mathrm{SD}$, Li R et al (2010). Mesolimbic dopamine reward system hypersensitivity in individuals with psychopathic traits. Nat Neurosci 13: 419-421.

Camara E, Kramer UM, Cunillera T, Marco-Pallares J, Cucurell D, Nager W et al (2010). The effects of COMT (Val108/158Met) and DRD4 (SNP-521) dopamine genotypes on brain activations related to valence and magnitude of rewards. Cereb Cortex 20: 1985-1996.

Carelli RM, Wightman RM (2004). Functional microcircuitry in the accumbens underlying drug addiction: insights from real-time signaling during behavior. Curr Opin Neurobiol 14: 763-768.

Chau DT, Roth RM, Green AI (2004). The neural circuitry of reward and its relevance to psychiatric disorders. Curr Psychiatry Rep 6: 391-399.

Chen F, Lawrence AJ (2003). The effects of antidepressant treatment on serotonergic and dopaminergic systems in FawnHooded rats: a quantitative autoradiography study. Brain Res $\mathbf{9 7 6}$ : 22-29.

Davis KL, Kahn RS, Ko G, Davidson M (1991). Dopamine in schizophrenia: a review and reconceptualization. Am J Psychiatry 148: $1474-1486$.

Delgado PL (2006). Serotonin noradrenaline reuptake inhibitors: new hope for the treatment of chronic pain. Int J Psychiatry Clin Pract 10(Suppl 2): 16-21.

Dreher JC, Kohn P, Kolachana B, Weinberger DR, Berman KF (2009). Variation in dopamine genes influences responsivity of the human reward system. Proc Natl Acad Sci USA 106: 617-622.

Dreher JC, Meyer-Lindenberg A, Kohn P, Berman KF (2008). Agerelated changes in midbrain dopaminergic regulation of the human reward system. Proc Natl Acad Sci USA 105: 15106-15111.
Fusar-Poli P, Meyer-Lindenberg A (2013). Striatal presynaptic dopamine in schizophrenia, part II: meta-analysis of [(18)F/(11) C]-DOPA PET studies. Schizophr Bull 39: 33-42.

Graf H, Metzger CD, Walter M, Abler B (2016). Serotonergic antidepressants decrease hedonic signals but leave learning signals in the nucleus accumbens unaffected. Neuroreport 27: $18-22$.

Greer SM, Goldstein AN, Knutson B, Walker MP (2016). A genetic polymorphism of the human dopamine transporter determines the impact of sleep deprivation on brain responses to rewards and punishments. J Cogn Neurosci 28: 803-810.

Gunn RN, Lammertsma AA, Hume SP, Cunningham VJ (1997). Parametric imaging of ligand-receptor binding in PET using a simplified reference region model. Neuroimage 6: 279-287.

Haber SN, Behrens TE (2014). The neural network underlying incentive-based learning: implications for interpreting circuit disruptions in psychiatric disorders. Neuron 83: 1019-1039.

Hagele C, Schlagenhauf F, Rapp M, Sterzer P, Beck A, Bermpohl F et al (2015). Dimensional psychiatry: reward dysfunction and depressive mood across psychiatric disorders. Psychopharmacology 232: 331-341.

Halldin C, Erixon-Lindroth N, Pauli S, Chou YH, Okubo Y, Karlsson $\mathrm{P}$ et al (2003). [(11)C]PE2I: a highly selective radioligand for PET examination of the dopamine transporter in monkey and human brain. Eur J Nucl Med Mol Imaging 30: 1220-1230.

Hirth N, Meinhardt MW, Noori HR, Salgado H, Torres-Ramirez O, Uhrig $S$ et al (2016). Convergent evidence from alcoholdependent humans and rats for a hyperdopaminergic state in protracted abstinence. Proc Natl Acad Sci USA 113: 3024-3029.

Hommer DW, Bjork JM, Gilman JM (2011). Imaging brain response to reward in addictive disorders. Ann N Y Acad Sci 1216: $50-61$.

Ikemoto S (2007). Dopamine reward circuitry: two projection systems from the ventral midbrain to the nucleus accumbensolfactory tubercle complex. Brain Res Rev 56: 27-78.

Insel T, Cuthbert B, Garvey M, Heinssen R, Pine DS, Quinn K et al (2010). Research domain criteria (RDoC): toward a new classification framework for research on mental disorders. Am J Psychiatry 167: 748-751.

Juckel G, Schlagenhauf F, Koslowski M, Filonov D, Wustenberg T, Villringer A et al (2006). Dysfunction of ventral striatal reward prediction in schizophrenic patients treated with typical, not atypical, neuroleptics. Psychopharmacology (Berl) 187: 222-228.

Kapur S (2003). Psychosis as a state of aberrant salience: a framework linking biology, phenomenology, and pharmacology in schizophrenia. Am J Psychiatry 160: 13-23.

Karila L, Leroy C, Dubol M, Trichard C, Mabondo A, Marill C et al (2016). Dopamine transporter correlates and occupancy by modafinil in cocaine-dependent patients: a controlled study with high-resolution PET and $\left[{ }^{11} \mathrm{C}\right]-\mathrm{PE} 2 \mathrm{I}$. Neuropsychopharmacology 41(9): 2294-2302.

Kim C-E, Lee M-H, Lee P-G, Choe W-S, Pyo S-J (2004). Correlation between psychopathology and dopamine transporter density in striatum before and after taking olanzapine assessed with IPTSPECT in first episode schizophrenia. Korean J Psychopharmacol 15: 8 .

Knutson B, Adams CM, Fong GW, Hommer D (2001a). Anticipation of increasing monetary reward selectively recruits nucleus accumbens. J Neurosci 21: RC159.

Knutson B, Fong GW, Adams CM, Varner JL, Hommer D (2001b). Dissociation of reward anticipation and outcome with eventrelated fMRI. Neuroreport 12: 3683-3687.

Knutson B, Westdorp A, Kaiser E, Hommer D (2000). FMRI visualization of brain activity during a monetary incentive delay task. Neuroimage 12: 20-27.

Kugaya A, Seneca NM, Snyder PJ, Williams SA, Malison RT, Baldwin RM et al (2003). Changes in human in vivo serotonin 
and dopamine transporter availabilities during chronic antidepressant administration. Neuropsychopharmacology 28: 413-420.

Lammertsma AA, Hume SP (1996). Simplified reference tissue model for PET receptor studies. Neuroimage 4(3 Pt 1): 153-158.

Lavalaye J, Knol RJ, de Bruin K, Reneman L, Janssen AG, Booij J (2000). [123I]FP-CIT binding in rat brain after acute and subchronic administration of dopaminergic medication. Eur J Nucl Med 27: 346-349.

Leroy C, Karila L, Martinot JL, Lukasiewicz M, Duchesnay E, Comtat C et al (2012). Striatal and extrastriatal dopamine transporter in cannabis and tobacco addiction: a high-resolution PET study. Addict Biol 17: 981-990.

Macoveanu J, Fisher PM, Haahr ME, Frokjaer VG, Knudsen GM, Siebner HR (2014). Effects of selective serotonin reuptake inhibition on neural activity related to risky decisions and monetary rewards in healthy males. Neuroimage 99: 434-442.

Malison RT, Best SE, Wallace EA, McCance E, Laruelle M, Zoghbi SS et al (1995). Euphorigenic doses of cocaine reduce [123I]betaCIT SPECT measures of dopamine transporter availability in human cocaine addicts. Psychopharmacology 122: 358-362.

McHugh PC, Buckley DA (2015). The structure and function of the dopamine transporter and its role in CNS diseases. Vitam Horm 98: 339-369.

Naranjo CA, Tremblay LK, Busto UE (2001). The role of the brain reward system in depression. Prog Neuropsychopharmacol Biol Psychiatry 25: 781-823.

Narendran R, Martinez D (2008). Cocaine abuse and sensitization of striatal dopamine transmission: a critical review of the preclinical and clinical imaging literature. Synapse 62: 851-869.

Nees F, Vollstadt-Klein S, Fauth-Buhler M, Steiner S, Mann K, Poustka L et al (2012). A target sample of adolescents and reward processing: same neural and behavioral correlates engaged in common paradigms? Exp Brain Res 223: 429-439.

Nielsen MO, Rostrup E, Wulff S, Bak N, Broberg BV, Lublin H et al (2012). Improvement of brain reward abnormalities by antipsychotic monotherapy in schizophrenia. Arch Gen Psychiatry 69: 1195-1204.

Pinsonneault JK, Han DD, Burdick KE, Kataki M, Bertolino A, Malhotra AK et al (2011). Dopamine transporter gene variant affecting expression in human brain is associated with bipolar disorder. Neuropsychopharmacology 36: 1644-1655.

Reith ME, Xu C, Zhang L, Coffey LL (1996). Translocation of dopamine and binding of WIN 35,428 measured under identical conditions in cells expressing the cloned human dopamine transporter. Naunyn Schmiedebergs Arch Pharmacol 354: 295-304.

Rominger A, Cumming P, Brendel M, Xiong G, Zach C, Karch S et al (2015). Altered serotonin and dopamine transporter availabilities in brain of depressed patients upon treatment with escitalopram: a [123 I]beta-CIT SPECT study. Eur Neuropsychopharmacol 25: 873-881.
Schott BH, Minuzzi L, Krebs RM, Elmenhorst D, Lang M, Winz OH et al (2008). Mesolimbic functional magnetic resonance imaging activations during reward anticipation correlate with rewardrelated ventral striatal dopamine release. J Neurosci 28: 14311-14319.

Schultz W (1997). Dopamine neurons and their role in reward mechanisms. Curr Opin Neurobiol 7: 191-197.

Seki C, Ito H, Ichimiya T, Arakawa R, Ikoma Y, Shidahara M et al (2010). Quantitative analysis of dopamine transporters in human brain using [11C]PE2I and positron emission tomography: evaluation of reference tissue models. Ann $\mathrm{Nucl} \mathrm{Med}$ 24: 249-260.

Sjoholm H, Bratlid T, Sundsfjord J (2004). 123I-beta-CIT SPECT demonstrates increased presynaptic dopamine transporter binding sites in basal ganglia in vivo in schizophrenia. Psychopharmacology 173: 27-31.

Stoy M, Schlagenhauf F, Sterzer P, Bermpohl F, Hagele C, Suchotzki $\mathrm{K}$ et al (2012). Hyporeactivity of ventral striatum towards incentive stimuli in unmedicated depressed patients normalizes after treatment with escitalopram. J Psychopharmacol 26: 677-688.

Volkow ND, Fowler JS, Wang GJ (2004). The addicted human brain viewed in the light of imaging studies: brain circuits and treatment strategies. Neuropharmacology 47(Suppl 1): 3-13.

Volkow ND, Wang GJ, Fischman MW, Foltin RW, Fowler JS, Abumrad NN et al (1997). Relationship between subjective effects of cocaine and dopamine transporter occupancy. Nature 386: 827-830.

Volkow ND, Wang GJ, Fowler JS, Tomasi D, Telang F (2011). Addiction: beyond dopamine reward circuitry. Proc Natl Acad Sci USA 108: 15037-15042.

Walter H, Kammerer H, Frasch K, Spitzer M, Abler B (2009). Altered reward functions in patients on atypical antipsychotic medication in line with the revised dopamine hypothesis of schizophrenia. Psychopharmacology 206: 121-132.

Weiland BJ, Heitzeg MM, Zald D, Cummiford C, Love T, Zucker RA et al (2014). Relationship between impulsivity, prefrontal anticipatory activation, and striatal dopamine release during rewarded task performance. Psychiatry Res 223: 244-252.

Weiland BJ, Zucker RA, Zubieta JK, Heitzeg MM (2016). Striatal dopaminergic reward response relates to age of first drunkenness and feedback response in at-risk youth. Addict Biol 22: 502-512.

Whitton AE, Treadway MT, Pizzagalli DA (2015). Reward processing dysfunction in major depression, bipolar disorder and schizophrenia. Curr Opin Psychiatry 28: 7-12.

Zhou Z, Zhen J, Karpowich NK, Law CJ, Reith ME, Wang DN (2009). Antidepressant specificity of serotonin transporter suggested by three LeuT-SSRI structures. Nat Struct Mol Biol 16: 652-657.

Supplementary Information accompanies the paper on the Neuropsychopharmacology website (http://www.nature.com/npp) 